\title{
Quasi-bound states in the continuum: a dynamical coupled-channel calculation of axial-vector charmed mesons
}

\author{
Susana Coitd* and George Rup ${ }^{\dagger}$ \\ Centro de Física das Interacções Fundamentais, Instituto Superior Técnico, \\ Technical University of Lisbon, P-1049-001 Lisboa, Portugal \\ Eef van Beveren \\ Centro de Física Computacional, Departamento de Física, \\ Universidade de Coimbra, P-3004-516 Coimbra, Portugal
}

(Dated: August 8, 2021)

\begin{abstract}
Masses and widths of the axial-vector charmed mesons $D_{1}(2420), D_{1}(2430), D_{s 1}(2536)$, and $D_{s 1}(2460)$ are calculated nonperturbatively in the Resonance-Spectrum-Expansion model, by coupling various open and closed meson-meson channels to the bare $J^{P}=1^{+} c \bar{q}(q=u, d)$ and $c \bar{s}$ states. The coupling to two-meson channels dynamically mixes and lifts the mass degeneracy of the spectroscopic ${ }^{3} P_{1}$ and ${ }^{1} P_{1}$ states, as an alternative to the usual spin-orbit splitting. Of the two resulting $S$-matrix poles in either case, one stays very close to the energy of the bare state, as a quasi-bound state in the continuum, whereas the other shifts considerably. This is in agreement with the experimental observation that the $D_{1}(2420)$ and $D_{s 1}(2536)$ have much smaller widths than one would naively expect. The whole pattern of masses and widths of the axial-vector charmed mesons can thus be quite well reproduced with only two free parameters, one of which being already strongly constrained by previous model calculations. Finally, predictions for pole positions of radially excited axial-vector charmed mesons are presented.

PACS numbers: 14.40.Lb, 13.25.Ft, 11.80.Gw, 11.55.Ds, 12.40.Yx,
\end{abstract}

\section{INTRODUCTION}

The axial-vector $(\mathrm{AV})$ charmed mesons $D_{1}(2420)$ and $D_{s 1}(2536)$ 1 have the puzzling feature that their decay widths are much smaller than one would expect on the basis of their principal $S$-wave decay modes. Namely, the $D_{1}(2420)$ decays to $D^{*} \pi$ (possibly also in a $D$ wave), with a phase space of more than $270 \mathrm{MeV}$, but has a total width of only $20-25 \mathrm{MeV} 1$. On the other hand, the $D_{s 1}(2536)$ decays to $D^{*} K$ in $S$ and $D$ wave with a phase space of about $30 \mathrm{MeV}$, resulting in an unknown tiny width $<2.3 \mathrm{MeV}$, limited by the experimental resolution [1. The discovery of the missing two AV charmed mesons, namely the very narrow $D_{s 1}(2460)$ and the very broad $D_{1}(2430)$, first observed by CLEO [2] and Belle [3, respectively, completed an even more confusing picture. While the tiny width of the $D_{s 1}(2460)$ can be easily understood, since this meson lies underneath its lowest Okubo-Zweig-Iizuka-allowed (OZIA) and isospin-conserving decay threshold, the huge $D_{1}(2430)$ width, in $D^{*} \pi$, is in sharp contrast with that of the $D_{1}(2420)$. Moreover, the $D_{s 1}(2460)$ lies $76 \mathrm{MeV}$ below the $D_{s 1}(2536)$, whereas the $D_{1}(2420)$ and $D_{1}(2430)$ are almost degenerate in mass, if one takes the central value of the latter resonance.

Quark potential models, with standard spin-orbit split-

\footnotetext{
*Electronic address: susana.coito@ist.utl.pt

${ }^{\dagger}$ Electronic address: george@ist.utl.pt

${ }^{\ddagger}$ Electronic address: eef@teor.fis.uc.pt
}

tings, fail dramatically in reproducing this pattern of masses. For instance, in the relativized quark model [4] the $c \bar{s}$ state that is mainly ${ }^{3} P_{1}$ comes out at $2.57 \mathrm{GeV}$, assuming the already then well-established $D_{s 1}(2536)$ to be mostly ${ }^{1} P_{1}$, though with a very large mixing between ${ }^{3} P_{1}$ and ${ }^{1} P_{1}$. Reference 4 similarly predicted a too high mass for the dominantly ${ }^{3} P_{1}$ state in the $c \bar{q}(q=u, d)$ sector, viz. $2.49 \mathrm{GeV}$. In the chiral quark model for heavy-light systems of Ref. [5], the result for the mainly ${ }^{3} P_{1} c \bar{q}$ state is also $2.49 \mathrm{GeV}$, while the discrepancy is even worse in the $c \bar{s}$ sector, with a prediction of $2.605 \mathrm{GeV}$ for the mostly ${ }^{3} P_{1}$ state, now with a small mixing in both sectors.

More recently and after the discovery of the $D_{s 1}(2460)$ (and $D_{1}(2430)$ ), chiral Lagrangians for heavy-light systems (see e.g. Refs. 66 9]) have been employed in order to understand the masses of the AV charmed mesons, in particular the mass splittings with respect to the vector (V) mesons with charm $D_{s}^{*}$ and $D^{*}$, respectively. Reference [7] analyzed in detail the curious experimental [1] observation that the AV-V mass difference is considerably larger in the charm-nonstrange sector than in the charmstrange one, which is not predicted by typical quark potential models [4, 5. The same discrepancy applies to the scalar-pseudoscalar mass difference in either sector 1, 7. In Ref. 7], the problem was tackled by calculating chiral loop corrections, but the result turned out to be exactly the opposite of what is needed to remove or alleviate the discrepancy.

An alternative approach to the AV charmed mesons is by trying to generate them as dynamical resonances in chiral unitary theory [10. Indeed, in the latter paper, describing AV mesons in other flavor sectors as 
well, several charmed resonances were predicted, including the $D_{1}(2420), D_{1}(2430), D_{s 1}(2536)$, and $D_{s 1}(2460)$, with reasonable results, though the $c \bar{q}$ states came out about $100 \mathrm{MeV}$ off. However, dynamical generation of mesonic resonances, including the ones that are commonly thought to be of a normal quark-antiquark type, may give rise to interpretational difficulties, besides predicting several genuinely exotic and so far unobserved states 10. Dynamically generated AV charmed as well as bottom mesons can be found in Ref. [11, too.

Finally, in Ref. 12 a coupled-channel calculation of positive-parity $c \bar{s}$ and $b \bar{s}$ was carried out in a chiral quark model, similar to our approach in its philosophy, and with results for the $D_{s 1}(2536)$ and $D_{s 1}(2460)$ close to the present ones (also see below).

\section{RESONANCE-SPECTRUM EXPANSION}

In the present paper, we employ the ResonanceSpectrum Expansion (RSE) to describe the AV charmed mesons. The RSE model has been developed for mesonmeson (MM) scattering in non-exotic channels, whereby the intermediate state is described via an infinite tower of $s$-channel $q \bar{q}$ states 13 . For the spectrum of the latter, in principle any confinement potential can be employed, but in practical applications, a harmonic oscillator (HO) with constant frequency has been used, with excellent results 13 . Recent applications of the RSE concern the $\phi(2170)$ [14 and $X(3872)$ [15] resonances.

In order to account for the two possible spectroscopic channels ${ }^{3} P_{1}$ and ${ }^{1} P_{1}$ contributing to a $J^{P}=1^{+}$state with undefined $C$-parity, we couple both $q \bar{q}$ channels to the most important meson-meson channels. The resulting fully off-energy-shell RSE $T$ matrix reads [13 15]

$$
\begin{aligned}
& T_{i j}^{\left(L_{i}, L_{j}\right)}\left(p_{i}, p_{j}^{\prime} ; E\right)=-2 \lambda^{2} \sqrt{\mu_{i} p_{i} r_{0}} j_{L_{i}}^{i}\left(p_{i} r_{0}\right) \times \\
& \sum_{m=1}^{N} \mathcal{R}_{i m}\left\{[\mathbb{1}-\Omega \mathcal{R}]^{-1}\right\}_{m j} j_{L_{j}}^{j}\left(p_{j}^{\prime} r_{0}\right) \sqrt{\mu_{j} p_{j}^{\prime} r_{0}}
\end{aligned}
$$

with the diagonal loop function

$$
\Omega_{i j}\left(k_{j}\right)=-2 i \lambda^{2} \mu_{j} k_{j} r_{0} j_{L_{j}}^{j}\left(k_{j} r_{0}\right) h_{L_{j}}^{(1) j}\left(k_{j} r_{0}\right) \delta_{i j},
$$

and the RSE propagator

$$
\mathcal{R}_{i j}(E)=\sum_{S=0,1} \sum_{n=0}^{\infty} \frac{g_{(S, n)}^{i} g_{(S, n)}^{j}}{E-E_{n}^{(S)}}
$$

Here, $\lambda$ is an overall coupling, $r_{0}$ is the average distance for decay via ${ }^{3} P_{0}$ quark-pair creation, $E_{n}^{(S)}$ is the discrete energy of the $n$-th recurrence in the $q \bar{q}$ channel with spin $S, g_{(S, n)}^{i}$ is the corresponding coupling to the $i$-th MM channel, $\mu_{i}$ the reduced mass for this channel, $p_{i}$ the off-shell relative momentum, $L_{i}$ the orbital angular momentum, and $j_{L_{i}}^{i}$ and $h_{L_{j}}^{(1) j}\left(k_{j} r_{0}\right)$ the spherical
Bessel and Hankel functions of the first kind, respectively. Note that $\mu_{i}, p_{i}$, and the on-energy-shell relative momentum $k_{i}$ are defined relativistically. Also notice that the infinite sum over the higher recurrences converges very fast, so that it can be truncated after 20 terms in practical calculations. The $S$ matrix is finally given by $S_{i j}^{\left(L_{i}, L_{j}\right)}(E)=1+2 i T_{i j}^{\left(L_{i}, L_{j}\right)}\left(k_{i}, k_{j} ; E\right)$.

\section{OZI-ALLOWED CHANNELS FOR AV CHARMED MESONS}

Now we describe the physical AV charmed resonances by coupling bare ${ }^{3} P_{1}$ and ${ }^{1} P_{1} c \bar{q}, c \bar{s}$ channels to all OZI-allowed ground-state pseudoscalar-vector $(\mathrm{PV})$ and vector-vector (VV) channels. It is true that there are also relevant pseudoscalar-scalar (PS) channels (in $P$-wave), most notably $D f_{0}(600)$ and $D_{0}^{*}(2400) \pi$ [1] in the $\mathrm{AV} c \bar{q}$ case, and $D K_{0}^{*}(800)$ for $c \bar{s}$. These will contribute to the observed [1] $D \pi \pi$ and $D \pi K$ decay modes, respectively. Now, we have recently developed [15] an algebraic procedure to deal with resonances in asymptotic states whilst preserving unitarity. However, the huge widths of the $D_{0}^{*}(2400), f_{0}(600)$, and $K_{0}^{*}(800)$ resonances may lead to fine sensitivities that will tend to obscure the point we want to make, apart from the fact that there will also be nonresonant contributions to the $D \pi \pi$ and $D \pi K$ final

TABLE I: Included meson-meson channels for $D_{1}(2420)$ and $D_{1}(2430)$, with ground-state couplings squared [16], orbital angular momenta, and thresholds in $\mathrm{MeV}$. For $\eta$ and $\eta^{\prime}$, a pseudoscalar mixing angle of $37.3^{\circ}$ [14] is used.

\begin{tabular}{ccccc}
\hline \hline Channel & $\left(\tilde{g}_{(S=1, n=0)}^{i}\right)^{2}$ & $\left(\tilde{g}_{(S=0, n=0)}^{i}\right)^{2}$ & $L$ & Threshold \\
\hline$D^{*} \pi$ & 0.02778 & 0.01389 & 0 & 2146 \\
$D^{*} \pi$ & 0.03472 & 0.06944 & 2 & 2146 \\
$D^{*} \eta$ & 0.00524 & 0.00262 & 0 & 2556 \\
$D^{*} \eta$ & 0.00655 & 0.01310 & 2 & 2556 \\
$D_{s}^{*} K$ & 0.01852 & 0.00926 & 0 & 2608 \\
$D_{s}^{*} K$ & 0.02315 & 0.04630 & 2 & 2608 \\
$D \rho$ & 0.02778 & 0.01389 & 0 & 2643 \\
$D \rho$ & 0.03472 & 0.06944 & 2 & 2643 \\
$D \omega$ & 0.00926 & 0.00463 & 0 & 2650 \\
$D \omega$ & 0.01157 & 0.02315 & 2 & 2650 \\
$D^{*} \rho$ & 0 & 0.01389 & 0 & 2784 \\
$D^{*} \rho$ & 0.01042 & 0.06944 & 2 & 2784 \\
$D^{*} \omega$ & 0 & 0.00463 & 0 & 2791 \\
$D^{*} \omega$ & 0.03472 & 0.02315 & 2 & 2791 \\
$D_{s} K^{*}$ & 0.01852 & 0.00926 & 0 & 2862 \\
$D_{s} K^{*}$ & 0.02315 & 0.04630 & 2 & 2862 \\
$D^{*} \eta^{\prime}$ & 0.00402 & 0.00201 & 0 & 2996 \\
$D^{*} \eta^{\prime}$ & 0.00502 & 0.01004 & 2 & 2996 \\
$D_{s}^{*} K^{*}$ & 0 & 0.00926 & 0 & 3006 \\
$D_{s}^{*} K^{*}$ & 0.06944 & 0.04630 & 2 & 3006 \\
\hline \hline
\end{tabular}


TABLE II: As Table【, but now for $D_{s 1}(2536)$ and $D_{s 1}(2460)$.

\begin{tabular}{ccccc}
\hline \hline Channel & $\left(\tilde{g}_{(S=1, n=0)}^{i}\right)^{2}$ & $\left(\tilde{g}_{(S=0, n=0)}^{i}\right)^{2}$ & L & Threshold \\
\hline$D^{*} K$ & 0.03704 & 0.01852 & 0 & 2504 \\
$D^{*} K$ & 0.04630 & 0.09259 & 2 & 2504 \\
$D_{s}^{*} \eta$ & 0.00803 & 0.00402 & 0 & 2660 \\
$D_{s}^{*} \eta$ & 0.01004 & 0.02009 & 2 & 2660 \\
$D K^{*}$ & 0.03704 & 0.01852 & 0 & 2761 \\
$D K^{*}$ & 0.04630 & 0.09259 & 2 & 2761 \\
$D^{*} K^{*}$ & 0 & 0.01852 & 0 & 2902 \\
$D^{*} K^{*}$ & 0.01389 & 0.09259 & 2 & 2902 \\
$D_{s} \phi$ & 0.01852 & 0.00926 & 0 & 2988 \\
$D_{s} \phi$ & 0.02315 & 0.04630 & 2 & 2988 \\
$D_{s}^{*} \eta^{\prime}$ & 0.01048 & 0.00524 & 0 & 3069 \\
$D_{s}^{*} \eta^{\prime}$ & 0.01310 & 0.02621 & 2 & 3069 \\
$D_{s}^{*} \phi$ & 0 & 0.00926 & 0 & 3132 \\
$D_{s}^{*} \phi$ & 0.06944 & 0.04630 & 2 & 3132 \\
\hline \hline
\end{tabular}

states. So we restrict ourselves to the open and closed $\mathrm{PV}$ and VV channels in the present investigation, but we shall further discuss this issue below. The here included channels for $c \bar{q}$ and $c \bar{s}$ are given in Tables I] and[II, respectively, together with the corresponding orbital angular momenta, threshold energies, and ground-state couplings squared $\left(\tilde{g}_{(S=1(0), n=0}^{i}\right)^{2}$, where $S=1(0)$ refers to the ${ }^{3} P_{1}\left({ }^{1} P_{1}\right)$ quark-antiquark component. In Appendix A we show in more detail how the ground-state coupling constants in Tables $\mathrm{I}$ and II depend on the isospin and $J^{P C}$ quantum numbers of the various meson-meson channels. The latter squared couplings, computed in the very general framework of Ref. [16, must be multiplied by $(n+1) / 4^{n}$ for $L=0$ and by $(2 n / 5+1) / 4^{n}$ for $L=2$, so as to obtain the couplings for the radial recurrences $n$ in the RSE sum of Eq. (3). Note that the scheme of Ref. [16] employs overlaps of $\mathrm{HO}$ wave functions for the original $q \bar{q}$ pair, the ${ }^{3} P_{0} q \bar{q}$ pair created out of the vaccuum, and the outgoing mesons. This allows to rigorously calculate the coupling constants of all excited states as well, in contrast with approaches using combinations of ClebschGordan coefficients only. Nevertheless, our ground-state couplings are identical to the usual ones in practically all situations, including the present one. Finally, a subthreshold suppression of closed channels is used just as in Ref. [14.

The energies of the bare AV $c \bar{q}$ and $c \bar{s}$ states we determine, as in previous work (see e.g. Refs. [14, 15), from an $\mathrm{HO}$ spectrum. The corresponding constant oscillator frequency and the constituent masses of the charmed, strange, and nonstrange quarks are also kept completely unchanged at the values $\omega=190 \mathrm{MeV}, m_{c}=1562 \mathrm{MeV}$, $m_{s}=508 \mathrm{MeV}$, and $m_{n}=406 \mathrm{MeV}$ [14, 15]. This yields masses of $2443 \mathrm{MeV}$ and $2545 \mathrm{MeV}$ for the bare $\mathrm{AV} c \bar{q}$ and $c \bar{s}$ states, respectively, which are very close to values found in typical single-channel quark models $[4,5$.

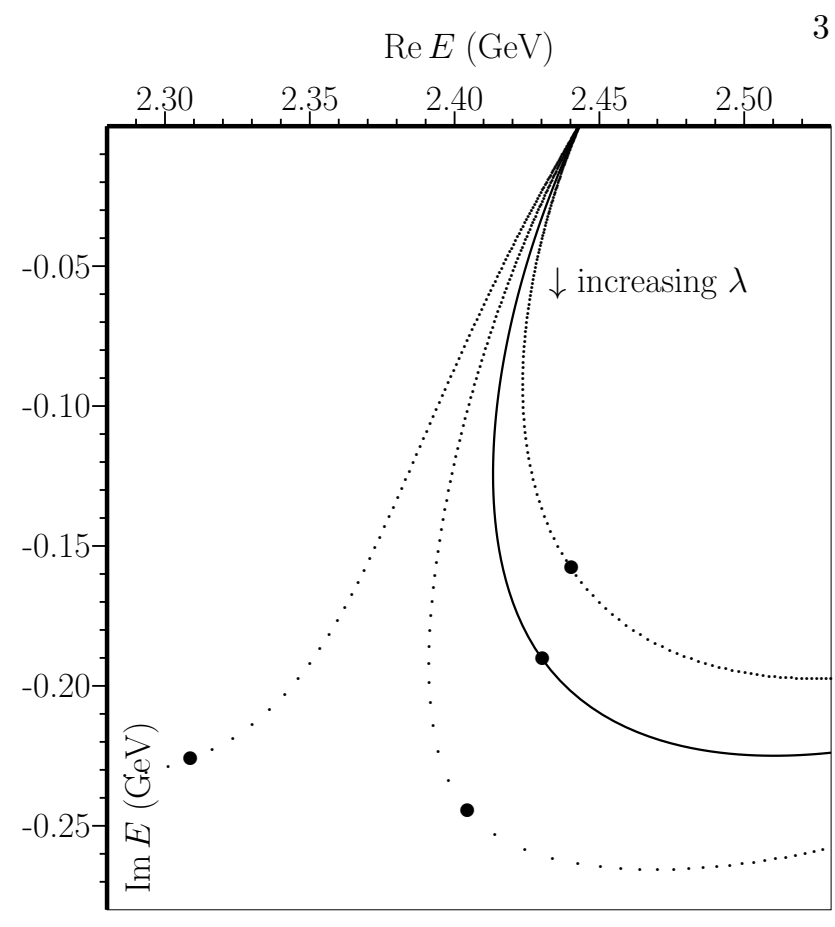

FIG. 1: $D_{1}(2430)$ pole trajectories as a function of $\lambda$, for $r_{0}=3.2-3.5 \mathrm{GeV}^{-1}$ (left to right). Solid curve and bullets correspond to $r_{0}=3.40 \mathrm{GeV}^{-1}$ and $\lambda=1.30$, respectively.

\section{QUASI-BOUND STATES IN THE CONTINUUM AND OTHER POLES}

Next we search for poles in the $S$ matrix. Starting with the $c \bar{q}$ case, we choose $r$ in the range 3.2 $3.5 \mathrm{GeV}^{-1}(0.64-0.70 \mathrm{fm})$, which is in between the values of $2.0 \mathrm{GeV}^{-1}$ [15] for an AV $c \bar{c}$ system and $4.0 \mathrm{GeV}^{-1}$ [14] for vector $s \bar{s}$ states. In Fig. 1] we plot several pole trajectories in the complex E plane as a function of the overall coupling $\lambda$. We see that this pole rapidly acquires a large imaginary part, whereas the real part changes considerably less, especially in the range $r_{0}=3.3-3.5 \mathrm{GeV}^{-1}$, making it a good candidate for the broad $D_{1}(2430)$ resonance. For $\lambda=1.30$ and $r_{0}=3.40 \mathrm{GeV}^{-1}$, the pole comes out at $(2430-i \times 191) \mathrm{MeV}$, being thus fine-tuned to the experimental mass and width [1]. However, there should be another pole in the $S$ matrix, since there are 2 quark-antiquark channels and more than $2 \mathrm{MM}$ channels. From the structure of the $T$ matrix in Eqs. (1) 3), one can algebraically show that the number of poles for each bare state is equal to $\min \left(N_{q \bar{q}}, N_{M M}\right)$, besides possible poles of a purely dynamical nature. Indeed, another pole originating from the bare $c \bar{q}$ state is encountered, with its trajectories depicted in Fig. 2. Quite remarkably, this pole moves very little, acquiring an imaginary part that is a factor 55 smaller than in the $D_{1}(2430)$ case, for the values $\lambda=1.30$ and $r_{0}=3.40 \mathrm{GeV}^{-1}$ (see solid lines and bullets in both figures). So this resonance, with a pole position of $(2439-i \times 3.5) \mathrm{MeV}$, almost decouples from the only open OZIA MM channel [17, viz. $D^{*} \pi$, representing a quasi-bound state in the continuum (QBSC) 


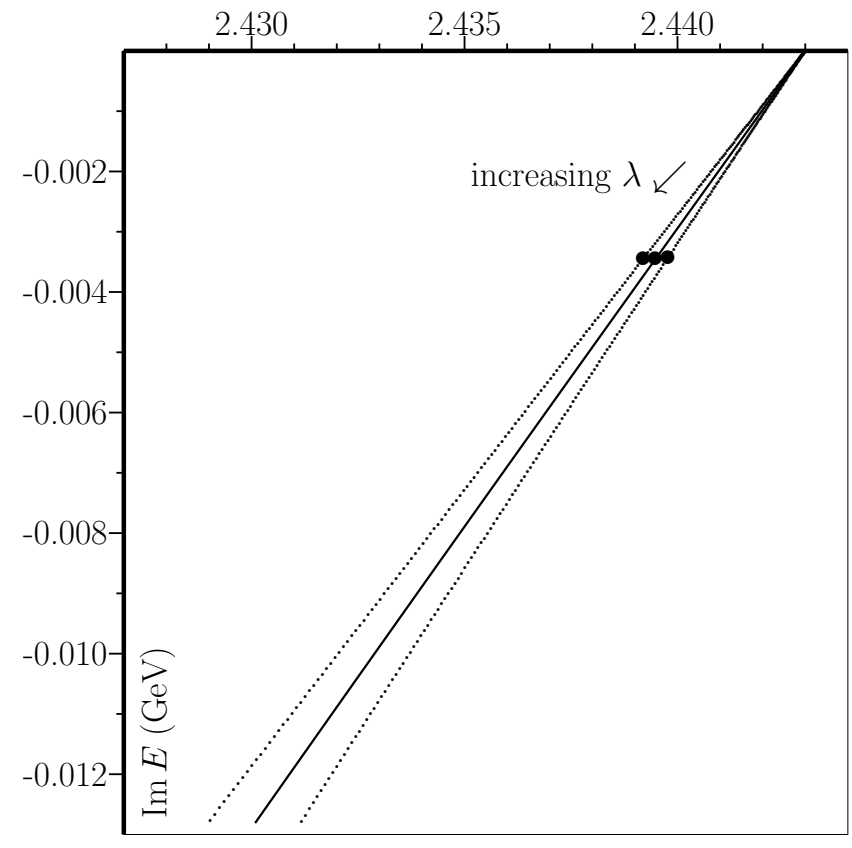

FIG. 2: $D_{1}(2420)$ pole trajectories as a function of $\lambda$, for $r_{0}=3.3-3.5 \mathrm{GeV}^{-1}$ (left to right). Solid curve and bullets correspond to $r_{0}=3.40 \mathrm{GeV}^{-1}$ and $\lambda=1.30$, respectively.

[11. Moreover, it is a good candidate for the $D_{1}(2420)$, though its width of roughly $7 \mathrm{MeV}$ is somewhat too small and its mass $16 \mathrm{MeV}$ too high. These minor discrepancies may be due to the neglect of the PS channels, with broad resonances in the final states, as suggested above.

Nevertheless, these encouraging results might be partly due to a fortuitous choice of the parameters $\lambda$ and $r_{0}$. Therefore, we now check the $c \bar{s}$ system, thereby scaling $r_{0}$ and $\lambda$ with the square root of the reduced quark mass (see Ref. [17, Eq. (13)), so as to respect flavor independence of our equations, which yields the $c \bar{s}$ values $r_{0}=3.12 \mathrm{GeV}^{-1}$ and $\lambda=1.19$. The ensuing $c \bar{s}$ pole trajectories are depicted in Fig. 3, but now for $r_{0}=3.12 \mathrm{GeV}^{-1}$ only. Thus, for $\lambda=1.19$, the strongly coupling state comes out at $2452 \mathrm{MeV}$, i.e., only $7.5 \mathrm{MeV}$ below the $D_{s 1}(2460)$ mass, with a vanishing width, as the pole ends up below the lowest OZIA channel. As for the $c \bar{s}$ QBSC, it indeed shifts very little from the bare state, settling at $(2540-i \times 0.7) \mathrm{MeV}$, i.e., only $5 \mathrm{MeV}$ above the $D_{s 1}(2536)$ mass, and having a width fully compatible with experiment [1].

Besides the above ground-state AV charmed mesons, the present model of course also predicts higher recurrences of these resonances. However, due caution is necessary so as to account for the most relevant open and closed decay channels at the relevant energy scales. Now, the first radially excited HO levels of the ${ }^{3} P_{1} /{ }^{1} P_{1} c \bar{n}$ and $c \bar{s}$ states lie at $2823 \mathrm{MeV}$ and $2925 \mathrm{MeV}$, respectively, which allows the corresponding resonances to be reasonably described by the channels included in Tables I.
III. Thus, we find again 4 poles, tabulated in Table III, together with those of the ground-state AV charmed

TABLE III: Poles of ground-state $(n=0)$ and first radiallyexcited $(n=1)$ AV charmed mesons. Parameters: $\lambda=1.30$ (1.19) and $r_{0}=3.40(3.12) \mathrm{GeV}^{-1}$, for $c \bar{q}(c \bar{s})$ states.

\begin{tabular}{ccc}
\hline \hline Quark Content Radial Excitation & Pole in $\mathrm{MeV}$ \\
\hline$c \bar{q}$ & 0 & $2439-i \times 3.5$ \\
$c \bar{q}$ & 0 & $2430-i \times 191$ \\
$c \bar{s}$ & 0 & $2540-i \times 0.7$ \\
$c \bar{s}$ & 0 & $2452-i \times 0.0$ \\
$c \bar{q}$ & 1 & $2814-i \times 7.8$ \\
$c \bar{q}$ & 1 & $2754-i \times 47.2$ \\
$c \bar{s}$ & 1 & $2915-i \times 6.7$ \\
$c \bar{s}$ & 1 & $2862-i \times 25.7$ \\
\hline \hline
\end{tabular}

mesons. For the radially excited states, we observe a similar pattern as for the ground states, namely two poles that remain close to the bare HO levels, whereas two other poles shift considerably. Note, however, that the difference is not as dramatic as in the $n=0$ case. This may be due to the fact that several decay channels are open now. As for a possible observation of the here predicted $2 P_{1}$ states, no experimental candidates have been reported so far. Namely, in the nearby $c \bar{q}$ mass region, the two listed 11 resonances $D(2600)$ and $D(2750)$ [1] both decay to $D^{*} \pi$ and $D \pi$, which excludes an AV assignment.

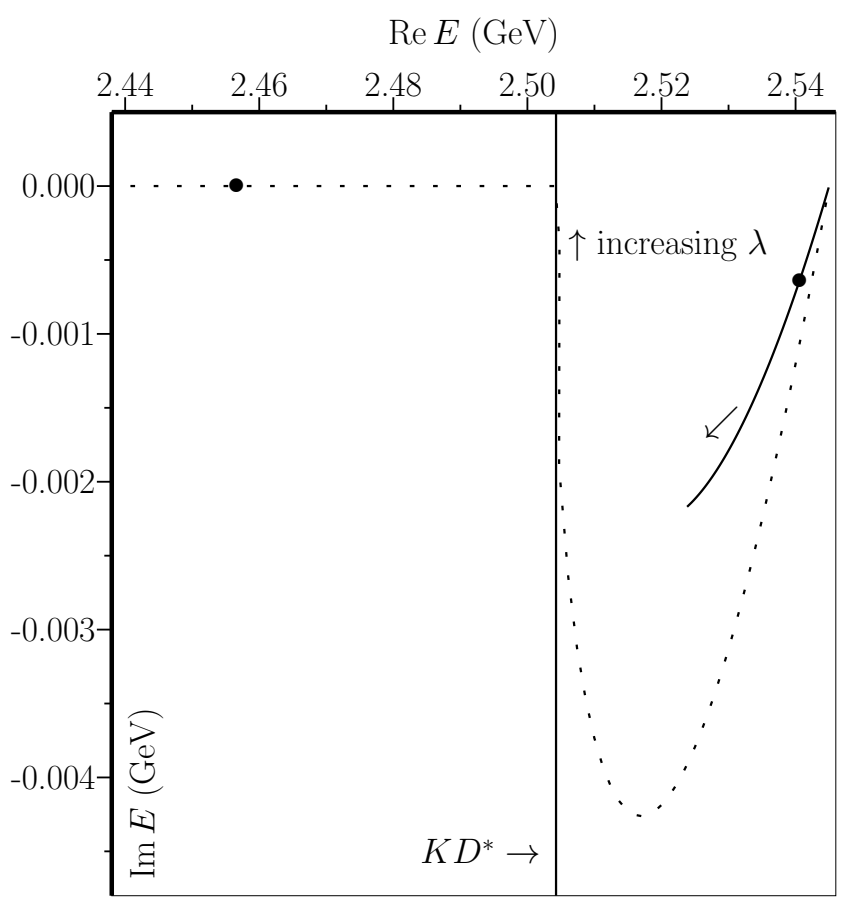

FIG. 3: $D_{s 1}(2460)$ (dashed) and $D_{s 1}(2536)$ (solid) pole trajectories as a function of $\lambda$, for $r_{0}=3.12 \mathrm{GeV}^{-1}$. Bullets correspond to $\lambda=1.19$; vertical line shows $D^{*} K$ threshold. 
Concerning the $c \bar{s}$ sector, the only listed [1] state around $2.8-2.9 \mathrm{GeV}$ is the $D_{s J}^{*}(2860)$ [18, with natural parity and so not an $\mathrm{AV}$, decaying to $D^{*} K$ and $D K$, which makes it a good candidate for the $2^{3} P_{2}$ state, possibly overlapped by the $2^{3} P_{0}[19$. Note that the lower of our two predicted $2 P_{1}$ resonances also practically coincides with the $D_{s J}^{*}(2860)$, both in mass and width. This may be a further indication that the $D_{s J}^{*}(2860)$ structure corresponds to more than one resonance only.

To conclude this section, we study - for the $c \bar{q}$ system - the dependence of the lowest-lying poles on the number of included quark-antiquark and MM channels. In Table IV, besides the $D_{1}(2420)$ and $D_{1}(2430)$ poles

TABLE IV: Poles of AV $c \bar{q}$ mesons, for different sets of included channels. Parameters: $\lambda=1.30, r_{0}=3.40 \mathrm{GeV}^{-1}$.

\begin{tabular}{cccc}
\hline \hline$c \bar{q}$ channels & MM channels & Pole $1(\mathrm{MeV})$ & Pole $2(\mathrm{MeV})$ \\
\hline${ }^{3} P_{1}+{ }^{1} P_{1}$ & 20 & $2430-i \times 191$ & $2439-i \times 3$ \\
${ }^{3} P_{1}+{ }^{1} P_{1}$ & 2 & $2402-i \times 36$ & $2441-i \times 1$ \\
${ }^{3} P_{1}+{ }^{1} P_{1}$ & 1 & $2431-i \times 39$ & - \\
${ }^{3} P_{1}$ & 20 & $2409-i \times 65$ & - \\
${ }^{1} P_{1}$ & 20 & $2425-i \times 96$ & - \\
\hline \hline
\end{tabular}

resulting from the full calculation, with the $20 \mathrm{MM}$ channels from Table I] we first give the pole positions for the cases that only $2\left(D^{*} \pi, L=0,2\right)$ or $1\left(D^{*} \pi, L=0\right)$ MM channels are included. The last two poles then correspond to calculations with the full $20 \mathrm{MM}$ channels but only one quark-antiquark channel, viz. ${ }^{3} P_{1}$ or ${ }^{1} P_{1}$. Notice that only one pole is found when the number of quarkantiquark or MM channels is equal to 1. This confirms our above conjecture that the number of poles for each bare $\mathrm{HO}$ level is given by $\min \left(N_{q \bar{q}}, N_{M M}\right)$.

\section{SUMMARY AND CONCLUSIONS}

In the foregoing, we have managed to rather accurately reproduce the masses and widths of the $D_{1}(2420)$, $D_{1}(2430), D_{s 1}(2536)$, and $D_{s 1}(2460)$ with only 2 free parameters, one of which is already constrained by previous model calculations, as well as by reasonable estimates for the size of these mesons. Crucial is the approximate decoupling from the continuum of one combination of ${ }^{3} P_{1}$ and ${ }^{1} P_{1}$ components, which amounts to a mixing angle close to $35^{\circ}$. Namely, if we express a QBSC as $|\mathrm{QBSC}\rangle=-\sin \theta\left|{ }^{3} P_{1}\right\rangle+\cos \theta\left|{ }^{1} P_{1}\right\rangle$, it decouples from the $L=0 D^{*} \pi$ channel (for $c \bar{q}$ ) or $D^{*} K$ channel (for $c \bar{s}$ ), if $\theta=\arccos \sqrt{2 / 3} \approx 35.26^{\circ}$ (see Tables I. II. Inclusion of the other, practically all closed, channels apparently changes the picture only slightly in our formalism. This result is in full agreement with the findings in Ref. [12]. However, in the present approach this particular mixing 20. comes out as a completely dynamical result, and is not chosen by us beforehand. Moreover, the bare-mass degeneracy of ${ }^{3} P_{1}$ and ${ }^{1} P_{1}$ states is adequately lifted via the decay couplings in Tables $\mathrm{I}$ and $\mathrm{I}$. dispensing with the usual $\vec{S} \cdot \vec{L}$ splitting. Also note that the occurrence of (approximate) bound states in the continuum for AV charmed mesons had already been conjectured by two of us [17, based on more general arguments.

The puzzling discrepancy between the $\mathrm{AV}-\mathrm{V}$ mass splittings in the $c \bar{q}$ and $c \bar{s}$ sectors is resolved in our calculation by dynamical, nonperturbative coupled-channel effects. A similar phenomenon we have observed before 21 for the $D_{0}^{*}(2300-2400)$ [1] resonance, and may be related to an effective Adler-type zero 22] in the $D^{*} \pi$ and $D \pi$ channels in the AV and scalar $c \bar{n}$ cases, respectively, owing to the small pion mass.

Summarizing, we have reproduced the whole pattern of masses and widths of the AV charmed mesons dynamically, by coupling the most important open and closed two-meson channels to bare $c \bar{q}$ and $c \bar{s}$ states containing both ${ }^{3} P_{1}$ and ${ }^{1} P_{1}$ components. The dynamics of the coupled-channel equations straightforwardly leads to one pair of strongly shifted states and another pair of QBSCs. Ironically, the state that shifts most in mass, namely the $D_{s 1}(2460)$, ends up as the narrowest resonance. This emphasizes the necessity [23] to deal with unquenched meson spectroscopy in a fully nonperturbative framework.

One might argue that these conclusions will depend on the specific model employed. Admittedly, our numerical results could change somewhat if slightly different bare masses for the AV charmed mesons were chosen, non- $S$ wave decay channels were included as well, or a different scheme was used to calculate the decreasing couplings of the higher recurrences. Nevertheless, we are convinced the bulk of our results will not change, most notably the appearance of QBSCs and the large shifts of their partner states, as the almost inevitable consequence of exact nonperturbative coupled-channel dynamics.

\section{Acknowledgments}

This work was supported in part by the Fundação para a Ciência e a Tecnologia of the Ministério da Ciência, Tecnologia e Ensino Superior of Portugal, under contract CERN/FP/116333/2010 and grant SFA-2-91/CFIF.

\section{Appendix A: Three-meson couplings}

The ground-state couplings in Tables $\mathrm{I}$ and II are obtained by multiplying the isospin recouplings given in Table $\sqrt{\mathrm{V}}$ with the $J^{P C}$ couplings in Table $\mathrm{VI}$ for an OZIA process $M_{A} \rightarrow M_{B}+M_{C}$ based on ${ }^{3} P_{0} q \bar{q}$ creation [16]. For clarity, we represent here all couplings by rational numbers. Note that $\eta_{n}$ and $\eta_{s}$ in Table $\mathrm{V}$ stand for the pseudoscalar $I=0$ states $(u \bar{u}+d \bar{d}) / \sqrt{2}$ and $s \bar{s}$, respectively. Then, we get the couplings to the physical $\eta$ and $\eta^{\prime}$ mesons by applying a mixing angle - in the flavor basis - of $41.2^{\circ}$, as in Ref. 21, 2nd paper. For the $\omega$ and $\phi$ we assume ideal mixing. 
TABLE V: Squared isospin recouplings for the 3-meson process $M_{A} \rightarrow M_{B}+M_{C}$, with $M_{A}=c \bar{s}$ or $c \bar{q}$.

\begin{tabular}{cccc}
\hline \hline$M_{A}$ & $M_{B}$ & $M_{C}$ & $g_{I}^{2}$ \\
\hline$D_{s 1}$ & $D_{s}, D_{s}^{*}$ & $\eta_{s}, \phi$ & $1 / 3$ \\
$D_{s 1}$ & $D, D^{*}$ & $K, K^{*}$ & $2 / 3$ \\
\hline$D_{1}$ & $D_{s}, D_{s}^{*}$ & $K, K^{*}$ & $1 / 3$ \\
$D_{1}$ & $D, D^{*}$ & $\pi, \rho$ & $1 / 2$ \\
$D_{1}$ & $D, D^{*}$ & $\eta_{n}, \omega$ & $1 / 6$ \\
\hline \hline
\end{tabular}

TABLE VI: Squared ground-state coupling constants for the 3-meson process $M_{A} \rightarrow M_{B}+M_{C}$, with $J^{P C}\left(M_{A}\right)=1^{+ \pm}$, and $M_{A}, M_{B}$ belonging to the lowest pseudoscalar or vector nonet.

\begin{tabular}{cccccc}
\hline \hline$J^{P C}\left(M_{A}\right)$ & $J^{P C}\left(M_{B}\right)$ & $J^{P C}\left(M_{C}\right)$ & $L_{M_{B} M_{C}}$ & $S_{M_{B} M_{C}}$ & $g_{(n=0)}^{2}$ \\
\hline $1^{++}$ & $0^{-+}$ & $1^{--}$ & 0 & 1 & $1 / 18$ \\
$1^{++}$ & $0^{-+}$ & $1^{--}$ & 2 & 1 & $5 / 72$ \\
$1^{++}$ & $1^{--}$ & $1^{--}$ & 0 & 1 & 0 \\
$1^{++}$ & $1^{--}$ & $1^{--}$ & 2 & 2 & $5 / 24$ \\
$1^{+-}$ & $0^{-+}$ & $1^{--}$ & 0 & 1 & $1 / 36$ \\
$1^{+-}$ & $0^{-+}$ & $1^{--}$ & 2 & 1 & $5 / 36$ \\
$1^{+-}$ & $1^{--}$ & $1^{--}$ & 0 & 1 & $1 / 36$ \\
$1^{+-}$ & $1^{--}$ & $1^{--}$ & 2 & 1 & $5 / 36$ \\
\hline \hline
\end{tabular}

[1] K. Nakamura et al. [Particle Data Group], J. Phys. G 37, 075021 (2010).

[2] D. Besson et al. [CLEO Collaboration], Phys. Rev. D 68, 032002 (2003) [Erratum-ibid. D 75, 119908 (2007)].

[3] K. Abe et al. [Belle Collaboration], Phys. Rev. D 69, 112002 (2004).

[4] S. Godfrey and N. Isgur, Phys. Rev. D 32, 189 (1985).

[5] M. Di Pierro and E. Eichten, Phys. Rev. D 64, 114004 (2001).

[6] W. A. Bardeen, E. J. Eichten, and C. T. Hill, Phys. Rev. D 68, 054024 (2003).

[7] D. Becirevic, S. Fajfer, and S. Prelovsek, Phys. Lett. B 599, 55 (2004).

[8] P. Colangelo, F. De Fazio, and R. Ferrandes, Mod. Phys. Lett. A 19, 2083 (2004).

[9] T. Mehen and R. P. Springer, Phys. Rev. D 72, 034006 (2005).

[10] D. Gamermann and E. Oset, Eur. Phys. J. A 33, 119 (2007).

[11] F. K. Guo, P. N. Shen, and H. C. Chiang, Phys. Lett. B 647, 133 (2007).

[12] A. M. Badalian, Yu. A. Simonov, and M. A. Trusov, Phys. Rev. D 77, 074017 (2008).
[13] E. van Beveren and G. Rupp, Annals Phys. 324, 1620 (2009).

[14] S. Coito, G. Rupp, and E. van Beveren, Phys. Rev. D 80, 094011 (2009).

[15] S. Coito, G. Rupp, and E. van Beveren, Eur. Phys. J. C 71, 1762 (2011).

[16] E. van Beveren, Z. Phys. C 21, 291 (1984).

[17] E. van Beveren and G. Rupp, Eur. Phys. J. C 32, 493 (2004).

[18] B. Aubert et al. [BABAR Collaboration], Phys. Rev. D 80, 092003 (2009).

[19] E. van Beveren and G. Rupp, Phys. Rev. D 81, 118101 (2010).

[20] Z. Y. Zhou and Z. Xiao, arXiv:1105.6025 [hep-ph].

[21] E. van Beveren and G. Rupp, Phys. Rev. Lett. 91, 012003 (2003); 97, 202001 (2006).

[22] D. V. Bugg, Phys. Rept. 397, 257 (2004); G. Rupp, F. Kleefeld, and E. van Beveren, AIP Conf. Proc. 756, 360 (2005).

[23] K. P. Khemchandani, E. van Beveren, and G. Rupp, Prog. Theor. Phys. 125, 581 (2011). 